\title{
Préparation expérimentale d'une souche de Irypanosoma congolense (Broden). Chimiorésistance au Moranyl (Suramine sodique)
}

\author{
por J. P. BERSON
}

La chımiorésistance des germes constitue, à l'heure actuelle, l'écueil majeur au bon développement de la chimiothérapie ef de la chimioprévention en médecine humaine comme en médecine vétérinaire, et, plus particulièrement dans ce domaine; quand il s'agit de juguler les grandes épizóoties.

$\mathrm{Si}$ le problème a subi quelques éclaircissements en matière de bactériologie, on peut dire qu'en ce qui concerne les protozoaires ef spécialement les trypanosomes, il reste entier. En effet, à propos de ceux-ci les expérimentations et les «suppositions » tendant à élucider le phénomène de chimiorésistance sont nombreuses, mais peu de résultats ont jusqu'à présent permis de se faire une opinion valable.

Quelle que soit cependant l'orientation à donner aux recherches, il convient d'avoir comme point de départ une souche de trypanosomes chimiorésistanis à une médication donnée, l'idéal étant, bien entendu, d'avoir une ou plusieurs espèces de trypanosomes « polyrésistants » aux trypanocides électropositifs, électronégatifs et faiblement ionisés offerts par la chimie moderne.

Le début de tout travail en matière de chimiorésistance devant être la création d'une souche de référence chimiorésistante à un premier médicament, on a exposé, dans les lignes qui suivent, la méthode ayant permis d'obtenir une souche de Trypanosoma congolense chimiorésistante au Moranyl.

Rev. Elev. Méd. vét. Pays trop. 1962, 15, no 1.

Reçu pour publication : février 1963.
La résistance au Moranyl devant permettre dans un stade ultérieur d'accéder à la résistance aux Moranylates, et de là, par le phénomène des résistances croisées, d'obtenır finalement une sauche de T. congolense omnirésistante.

L'aspect pharmacodynamique et thérapeutique du Moranyl ayant déjà été envisagé d'une manière exhaustive dans un précédent travail $\left({ }^{*}\right)$ c'est donc l'expérimentation seule qui a été rapportée ici.

\section{MATÉRIEL ET MÉTHODE}

Les animaux d'expérience utilisés sont des souris blanches mâles et femelles pesant $20 \mathrm{~g}$ environ.

La souche de Trypanosoma congolense provient de l'Institut Pasteur de Paris, où elle a été entretenue sur cobayes et souris depuis plusieurs mois, et n'a pas eu de contact avec le médicament utilisé.

Le Moranyl a été gracieusement fourni par la société Spécia Rhône-Poulenc.

De nombreuses expérimentations préliminaires ont été réalisées en vue de déterminer les doses initiales de Moranyl à utiliser pour mettre en route le phénomène de chimiorésistance, et font l'objet d'une relation séparée. Dans une

(*) BERSON (J. P.). 1962. Contribution à l'étude de Tryponosoma congolense. Sa biologie et sa chimiorésisiance au Moranyl el d̀ t'Antrycide Pro-salt. Thèse de Doctorai Vétérinaire. Lyon. 
deuxième partie a été exposée une série de rechutes de l'infection, suivie de passages sur animaux neufs, ayant permis d'abtenir des trypanosomes chimiorésistants sur des souris traitées avec doses de Moranyl allant de 250 à $500 \mathrm{mg} / \mathrm{kg}$.

\section{RÉSULTATS}

La dose minimale pour la souris étant de 1,35 gamma/gramme, il a été décidé de commencer le premier trastement de la première souris parasitée par une dose inférieure, soit 0,5 gamma/gramme.

Le 28-9 la souris (IPS 5 F Sm) reçoit cette dose, après avoir été inoculée le 13-9 avec la souche de trypanosomes normaux. En dépit du traitement les parasites se multiplient, ef l'infection parvient au stade maximum $(++++$ suivant notre cotation). Le 29-9, 2-10, 3-10 ef 4-10 la souris reçoit 2 gamma/gramme de Moranyl sans montrer de modification du taux de sa parasitémie. Bien au contraire le 5-10 l'administration de $2,5 \mathrm{gamma} / \mathrm{gramme}$ semble relancer l'infection.

Le 2-10 une souris ( $\times 3 \mathrm{~b} M$ COD-CrOG) est inoculée à partir de la précédente, les trypanosomes sont alors résistants à 2 gamma. Dès que les parasıtes apparaissent dans le sang périphérique, la souris est traitée à $5 \mathrm{gamma} / \mathrm{gram}$ me le 6-10. L'infection parvient cependant' au stade ++++ . Du 9-10 au 13-10 est pratıquée une série de traitements d̀ $8,10,15,20,25 \mathrm{gamma}$ gramme, sans que le nombre des parasites diminue. Le 16-10, l'animal étant devenu négatif, on administre tout de même $20 \mathrm{gamma} / \mathrm{gramme}$, 27,5 le 17 et 10 le 20-10, contre toute attente l'infection finit par atteindre le stade $t++\dot{\alpha}$ ++++ le 23-10.

La souris reste positive jusqu'au 7-11 bien que les traitements soient multipliés : $20,25,27,5,30$, $32,5,35,40,45$ et 50 gamma/gramme.

Le 9-11, le sang de la souris est devenu négatif, répétant la même opération que le 16-10, $50 \mathrm{gamma} / \mathrm{gramme}$ de Moranyl sont injectés suivis de 50 le 10-11. Le 16-11, l'infection est revenue au stade ++ , et la souris reste positive. On peut dire que le 23-11 les trypanosomes, non seulement sont résistants ¿̀ 90 gamma, mais sans doute à une dose de trypanocide bien plus importante, si l'on tient compte de sa lente élimination.

Une autre souris ( $Z$ F Sm) inoculée le 5-10 avec la même souche de $T$. congolense devenue chimiorésistante à 4 gamma/gramme, reçoit en même temps 6 gamma/gramme de Moranyl. L'infection parvient à son degré maximum le 10-10 et reste d̀ ce niveau jusqu'au 23-10, malgré les traitements suivants : $12,10,15,15,15,20$, 25,30 , ef le dernier, 35 gamma/gramme, a lieu le 20-10. Le 23 , la parasitémie diminue pour revenir au stade ++ . Une succession de traitements à 37,5 gamma le 24-10 40 le 25-10, 45 le 26-10, 52,5 le 31-10 et 55 le 2-11 permettent à la parasitémie de se maintenir à son niveau maximum.

Isolés d'autres souris, mais obtenus dans les mêmes conditionș, des trypanosomes chimiorésistants à 150 gamma/gramme sont inoculés le 14-11 à une souris (IP S5 M COG). Les parasites apparaissent dans le sang le 17, et dès le 18-11 un traitement à 150 gamma-gramme est pratiqué. Du 18 au 24-11, on enregistre une légère augmentation de la parasitémie, et pendant ce laps de temps la souris reçoit $1090 \mathrm{gamma} / \mathrm{gramme}$ de Moranyl. Le 25, I'infection est toujours de type + , ce qui prouve qu'en l'espace de 7 jours la chimiorésistance s'est accrue de 50 gamma. La souris finit d'ailleurs par succomber du falt de l'accumulation d'u trypanocide.

Avec une autre souris (IP IO'M COD), l'augmentation du taux de la chimiorésistance a été du même ordre pendant le même temps.

Toutes les souris trypanosomées et traitées ont répondu d'une manière identıque, à savoir que l'inoculation infectante associée au traitement, stımule le départ de la,chimiorésistance. Cette dernı̀re semble être augmentée et entretenue par des trattements élevés et successifs. On doit cependant, remarquer que sl ceux-ci voisinent les doses toxiques pour la souris, il devient impossible de, conserver les animaux très longtemps en raison du phénomène d'accumulation.

C'est pourquoi et parallèlement aux observations signalées ci-dessus, il a été entrepris de créer la chimiorésistance au Moranyl en associant la méthode des rechutes à celle des passages successifs, suivant la méthode proposée par 
G. CHAUVIER à I'Institut d'Elevage et de Médecine vétérinaire des Pays tropicaux.

\section{OBTENTION DE CHIMIORÉSISTANCE PAR RECHUTES ET PASSAGES}

La souris (IP $10 \mathrm{M}$ CrOG) est inoculée le 13-10 avec le sang d'un rat positif au stade +. L'infection met douze jours pour arriver au maximum ce qui s'explique par le fait que les parasites inoculés à la souris sont plus «habitués» au rat, alors que les trypanosomes entretenus sur souris déclenchent ordinairement une parasitémie maximum en 4 à 5 jours. Le 25-10, on administre $5 \mathrm{gamma} / \mathrm{gramme}$ de Moranyl, puis 40 le 26 , ef 50 le 31 . Du 2 au 8-11 on pratique cinq traitements échelonnés de 60 à 120 gamma gramme. Avant le passage sur la souris (IP $10 \mathrm{~F}$ COG « $A »)$ il est procédé à deux autres traitements de 120 ef 130 gamma.

Inoculée le 13-11 la souris (IP 10 F COG «A») développe une parasitémie dont le maximum est atteint plus rapidement que chez la souris précédente. On admınistre deux traitements à 130 gamma/gramme le 17 et le 19/11. Son sang est alors «passé » sur la souris (IP $10 \mathrm{~F}$ COG «B»). En même temps que l'inoculation est effectuée dans le péritoine, 150 gamma de Moranyl par gramme sont injectés par la voie sous-cutanée. Sans doute du fait de l'augmentation trop rapide des doses de trypanocide, les trypanosomes semblent perdre peu à peu de leur pouvoir infectant puisque la parasıtémı ne dépasse pas le stade +. La souris reçoit $170 \mathrm{gam}$ ma le 23 et 190 le 31-11, à cette date son sang est «passé » à deux souris neuves.

La souris (IP $10 \mathrm{~F}$ COD) est inoculée le 1-12 avec des trypanosomes « théoriquement 》 résistanis à 190 gamma/gramme; par mesure de sécurité, un traitement à $170 \mathrm{gamma} / \mathrm{gramme}$ est associé à l'inoculation infectante. La parasitémie atteınt le stade +++ ; la souris meurt le 8-12 après avoir reçu $250 \mathrm{gamma} / \mathrm{gramme}$. Le jour même son sang est «passé » sur une souris neuve.

La souris (Mo F Sm) est inoculée le 7-12 avec le sang de la souris (IP $10 \mathrm{~F} \mathrm{COD),} \mathrm{elle} \mathrm{reçoit}$ en même temps par voie sous-cutanée 200 gamma/gramme de Moranyl. L'infection devient maximum le 12-12. Elle est alors traitée avec
250 , puis 275 gamma/gramme le 14-12. A ceite date l'animal est saigné et son sang est recueilli et conservé à la température de l'azote liquide (*).

Enfin la souris (Mo F COD) inoculée le 1-12 avec le sang de la souris.(IP $10 \mathrm{~F} \ll \mathrm{B} \gg)$ reçoit le même jour 170 gamma/gramme de Moranyl. Sa longévité est plus grande, semble-t-il, que celle des autres souris. Après trois trattements de 250,275 et 290 gamma/gramme son sang est également placé dans l'azote liquide, dans le but de conserver les trypanosomes devenus chimiorésistants.

\section{CONCLUSION}

II a été possible d'obtenir rapidement une souche de Trypanosoma congolense résistante à la dose léthale 50 de Moranyl pour la souris, soit 250 gamma/gramme.

Depuis le premier traitement à 5 gamma/ gramme, jusqu'au traitement de $250 \mathrm{gamma}$ suivi de rechute, il a fallu effectuer 4 passages en 45 ou 49 jours suivant l'anımal considéré.

Cette résistance a d'ailleurs été portée à $500 \mathrm{gamma} / \mathrm{gramme}$ avec 2 passages supplémentaires effectués en 15 jours.

Au cours de cette expérimentation, la plupart des passages ont été pratiqués en associant l'ınoculation infectante à une dose de trypanocide déterminée, suivant la technique préconisée par G. CHAUVIER.

Sans qu'll soit possible de porter un jugement définitif, il semble que dans les conditions de l'expérience, le Moranyl utilisé agissait comme un facteur stimulant le développement de l'infection, sans qu'il nous soit possible d'expliquer ou de discuter les constatations que nous avons faites.

Les souches isolées des différentes souris, ont été passées sur des rats, où elles se sont montrées également résistantes pour des doses de médicament équivalentes à celles utilisées chez la souris.

Institut d'Elevage ef de Médecine vétérinaire des Pays fropicaux.

(*) BERSON (J. P.). Utilisation du liquide Hanks pour la conservation de Trypanosoma congolense par le froid. Bull. Soc, Pathol. Exo. 1962, 55 (5) : 804-7. 


\section{SUMMARY}

The production experimentally of a strain of $T$. congolense chemoresistant to Moranyl

The author describes his method. In most passages the infective inoculum was associated with a calculated dose of trypanocide.

\section{RESUMEN}

Preparación experimental de una estirpe de Trypanosoma congolense (Broden) químicorresistente al Moranyl (Suramina sôdica)

El autor indica el método por él utilizado para obtener una estirpe de Trypanosoma congolense resistente al Moranyl.

Durante el transcurso de este experimento, la mayor parte de los pasos han sido procticados combinando la inoculaciôn infectante con una dosis determinada de tripanocida. 